\title{
Limit Theorems of Integrals with Respect to Vector Random Measures in Complete Paranormed Spaces
}

\author{
Renying Zeng \\ School of Mathematical Sciences, Chongqing Normal University, Chongqing, China
}

Email address:

renying.zeng@saskpolytech.ca

\section{To cite this article:}

Renying Zeng. Limit Theorems of Integrals with Respect to Vector Random Measures in Complete Paranormed Spaces. International Journal of Statistical Distributions and Applications. Vol. 3, No. 4, 2017, pp. 81-86. doi: 10.11648/j.ijsd.20170304.14

Received: September 7, 2017; Accepted: September 26, 2017; Published: November 15, 2017

\begin{abstract}
This paper studies random integral of the form $\int g d M$, where $f$ is a function taking value in a paranormed vector space $X$, and $M$ is an independent scattered vector random measure. Random integrals of this type are a natural generalization of random series with paranormed space valued coefficients. Some limit theorems of integrals with respect to vector random measures are proved.
\end{abstract}

Keywords: Paranormed Vector Space, Random Measure, Random Integral, Limit Theorem, Convergence in Probability

\section{Introduction}

The idea of random measures first appeared in Bochner's paper [1], and a variety of discussions followed [8, 10, 12, 15, $17,18,19,20]$. The aim of this article is to study random integral of the form $\int g d M$, where $g$ is a function taking value in a paranormed vector space $X$, and $M$ is an independent scattered vector random measure. Random integrals of this type are a generalization of random series with Banach space valued coefficients. It is well known that the asymptotic behavior of such series depends also on some geometric properties of the Banach space $X$ (or a metrizable space) $[3,4,6,8,16]$. On the other hand, the existence of certain bounded linear operators on appropriate function spaces which we call random integrals depends in general on a geometric structure of $X[12,18,19]$.

This paper defines and studies random integrals without any restriction in the geometry of $X$, devotes to the study of independently scattered vector random measures with emphasis on their convergence properties, presents convergence theorems of random integrals of the forms $\int g_{n} d M$ and $\int g d M$, where $g_{n}$ and $g$ are taking values in a paranormed vector space $X$, and $M_{n}$ and $M$ are $R^{1}$-valued random measures.

\section{Paranormed Spaces}

Let $K$ be the field of real numbers or the field of complex numbers, and $X$ be a vector space over $K$. A paranorm $\|\cdot\|$ is a function defined on $X$, satisfying

$$
\begin{gathered}
\|x\| \geq 0,\|x\|=0 \Leftrightarrow x=0 ; \\
\|x+y\| \leq\|x\|+\|y\|
\end{gathered}
$$

$\|-x\|=\|x\| ;$ and

$\lim _{n \rightarrow 0}\left\|\alpha_{n} x\right\|=0$, for any sequence $\left\{\alpha_{n}\right\} \subset K$;

$\lim _{n \rightarrow 0}\left\|\alpha x_{n}\right\|=0$, for any sequence $\left\{x_{n}\right\} \subset X$.

Since $\|x-y\| \leq\|x\|+\|y\|$, for $\forall x, y \in X,\|x-y\|$ defines a metric on $X$. $X$ equipped with the metric topology is said to be a paranormed space.

A paranormed space is said to be complete if every Cauchy sequence $\left\{x_{n}\right\}$ is convergent in $X$.

Any Banach space is a complete paranormed space. But the converse is not true.

A seminorm is function $q$ defined on $X$ and satsfying $q(x) \geq 0$;

$q(x+y) \leq q(x)+q(y)$; and

$q(\alpha x)=|\alpha| q(x)$ for any scalar $\alpha$.

From [2], one has the following Lemma 1.1. 
Lemma $1.1 \mathrm{X}$ is a complete paranormed space if and only if there is a family of continuous siminorms $Q=\left\{q_{n} ; n=1\right.$, $2, \ldots\}$ on $X$, such that

$$
q_{1}(x) \leq q_{2}(x) \leq \cdots \leq q_{n}(x) \leq \cdots, \forall x \in X .
$$

And the paranorm on $X$ can be given by

$$
\|x\|=\sum_{n=1}^{\infty} \frac{1}{2^{n}} \frac{q_{n}(x)}{1+q_{n}(x)}, \forall x \in X .
$$

For any sequence $\left\{x_{n}\right\} \subset X$, and $x \in X$, the following are equivalent

$$
\begin{aligned}
& \lim _{n \rightarrow \infty} x_{n}=x ; \\
& \lim _{n \rightarrow \infty}\left\|x_{n}-x\right\|=0 ; \text { or } \\
& \lim _{n \rightarrow \infty} q\left(x_{n}-x\right)=0, \forall q \in Q .
\end{aligned}
$$

\section{Random Integrals}

Let $(T, \Sigma)$ be a measurable space, and $(\Omega, \Gamma, P)$ be a probability space. A function

$$
M: \Sigma \rightarrow L_{0}(\Omega, \Gamma, P)
$$

is said to be an independently scattered random measure if for every pairwise disjoint sets $A_{n} \in \Sigma$, random variable $M\left(A_{n}\right) \quad$ are independent, $n=1, \quad 2, \quad \ldots, \quad$ and $M\left(\bigcup_{n=1}^{\infty} A_{n}\right)=\sum_{n=1}^{\infty} M\left(A_{n}\right)$.

Every independently scattered random measure $M$ can be decomposed into two independent and independently scattered random measure $M=M_{a}+M_{c}$, where $M_{a}$ is pure atomic, and $M_{c}$ is atomless.

Let $(T, \Sigma, \mu)$ be a finite measure space, and $v$ be an infinitely divisible distribution on $R^{1}$. The function

$$
M: \Sigma \rightarrow L_{0}(\Omega, \Gamma, P)
$$

is a random measure on $(T, \Sigma, \mu)$ generated by $v$ if $M$ is independently scattered and, for every $A \in \Sigma$,

$$
\mathscr{L}(M(A))=v^{* \mu(A)},
$$

where $v^{* p}$ is the $\mathrm{p}^{\text {th }}$ convolution power of $v$.

Daniell-Kolmogorov's Consistency Theorem implies the existence of a random measure on every measure space generated by every infinite divisible law.

In what follows we assume that $v$ is symmetric. From [3], we may write

$$
E \exp (i u M(A))=\exp [-\mu(A) K(u)],
$$

where

$$
K(u)=\frac{1}{2} \sigma^{2} u^{2}+\int_{-\infty}^{+\infty}(1-\cos u v) m(d v),
$$

while $m$ is a symmetric $\sigma$-finite measure on $R^{1}$ such that $M(\{0\})=0$ and $\int_{-\infty}^{+\infty} \min \left(1, v^{2}\right) m(d v)<\infty$. We write $M \cong\left[\sigma^{2}, m\right]$ if (1) and (2) hold.

In the sequel let $X$ be a paranormed space, $X^{\prime}$ be the topological dual space of $X$.

A function $g: T \rightarrow X$ is said to be a simple function if there exist pairwise disjoint measurable sets $B_{j} \in \Sigma$ and $x_{j} \in X(j=1$, $2, \ldots, k)$ such that

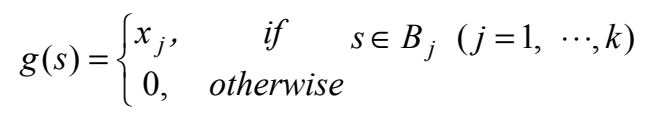

For every $A \in \Sigma$, we set

$$
\int_{A} g d M=\sum_{j=1}^{k} x_{j} M\left(B_{j} \cap A\right)
$$

the $\mu$-Integral of the simple function $g$ is defined as

$$
\int_{T} g(s) d \mu=\sum_{j=1}^{k} x_{j} \mu B_{j}
$$

Definition 3.1 A function $g: T \rightarrow X$ is said to be $M$ Integrable, if there exists a sequence of $\left\{g_{k}\right\}$ of simple functions from $T$ to $X$ such that

$$
\lim _{k \rightarrow \infty} g_{k}(s)=g(s), \quad \mu \text {-a.e. on } T
$$

i.e., there exists $E \in \Sigma$ and $\mu(E)=0$ such that $\lim _{k \rightarrow \infty} g_{k}(s)=g(s)$ for $s \in T \backslash A ;$ and for every $A \in \Sigma$, $\int_{A} g_{k} d M$ converges in probability.

If $g$ is $M$-integrable, we put

$$
\int_{A} g d M=P-\lim _{k \rightarrow \infty} \int_{A} g_{k} d M,
$$

then the symmetry and independence assumptions imply that for every seminorm $q \in Q$, every simple function $g: T \rightarrow X$, and every $A \in \Sigma$

$$
P\left\{q\left(\int_{A} g_{k} d M\right)>\varepsilon\right\} \leq P\left\{q\left(\int_{T} g_{k} d M\right)>\varepsilon\right\}, \varepsilon>0
$$

Hence condition (3) in the Definition 3.1 is equivalent to

$$
\int_{T} g_{k} d M \text { converges in probability. }
$$

Let $\mathscr{L}_{E}(M)$ denote the linear subspace of $L_{0}^{X}(T, \Sigma, \mu)$ consisting of all $M$-integrable functions. Similar to the proof of the Theorem in [5], $\mathscr{L}_{E}(M)$ is a complete metrizable vector space with the paranorm

$$
\|g\|_{M}=\int_{T} \min \{1,\|g\|\} \mu(d t)+E \min \left\{1,\left\|\int_{T} f d M\right\|\right\}
$$


A random variable $M \cong\left[\sigma^{2}, m\right]$ is said to be Poissonian if $\sigma^{2}=0$ and $M\left(R^{1}\right)<\infty$.

Proposition 3.1 If $M$ is a poissonian random measure on $(T, \Sigma, \mu)$, then

$$
\mathscr{L}_{E}(M)=L_{0}^{X}(T, \Sigma, \mu)
$$

Proof. Without loss of generality, we may assume that $M\left(R^{1}\right)>0$. For $u \geq 0$, set

$$
H(u)=\int \min \{u|v|, 1\} m(d v)
$$

then

$$
\begin{aligned}
& E \min \{u|M(A)|, 1\} \\
& =e^{-\mu(A) m(R)} \sum_{k=0}^{\infty} \frac{\mu^{k}(A)}{k !} \int_{-\infty}^{+\infty} \min \{u|v|, 1\} m^{* k}(d v) \\
& \leq H(u) \mu(A) .
\end{aligned}
$$

Therefore, for any simple function

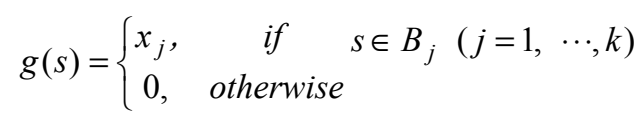

where $B_{j} \in \Sigma$ pairwise disjoint measurable sets, and for any seminorm $q$ in $Q$ we have

$$
\begin{aligned}
& E \min \left\{q\left(\int_{T} f d M\right), 1\right\} \\
& \leq \sum_{j=1}^{k} E \min \left\{q\left(x_{j}\right)\left|M\left(A_{j}\right)\right|, 1\right\} \\
& \leq \sum_{j=1}^{k} H\left(q\left(x_{j}\right)\right) \mu\left(A_{j}\right) \\
& \quad=\int_{T} H(q(f)) \mu(d t) .
\end{aligned}
$$

The proof is completed.

Proposition 3.2 Suppose that $E|M(T)|<\infty$. Then, there exists a constant $C_{M}$ such that for every simple function $g: T \rightarrow R^{1}$,

$$
E\left|\int_{T} g d M\right| \leq c_{M}\left(\int_{T} g^{2} d \mu\right)^{1 / 2}
$$

Proof. Suppose $M=M_{a}+M_{c}$, where $M_{a}$ is pure atomic, and $M_{c}$ is atomless.

Write $g(s)=\sum_{n=1}^{k} b_{n} \chi_{B_{n}}$, where $B_{n} \in \Sigma$ pairwise disjoint measurable sets, one has

$$
\begin{aligned}
& E\left|\int_{T} g d M_{a}\right| \\
& \leq\left(E\left|\int_{T} g^{2} d M_{a}\right|\right)^{1 / 2} \\
& =\left(\sum_{n=1}^{k} b_{n}^{2} E M_{a}^{2}\left(B_{n}\right)\right)^{1 / 2} \\
& =c_{0}\left(\int_{T} g^{2} d \mu \mid\right)^{1 / 2},
\end{aligned}
$$

where $c_{0}$ is a constant. And there exists a constant $c_{1}$ such that

$$
\begin{aligned}
& E\left|\int_{T} g d M_{c}\right| \\
& \leq\left(\sum_{n=1}^{k}\left|b_{j}\right| E M_{1}\left(B_{n}\right)\right)^{1 / 2} \\
& \leq c_{1} \int_{T}|g| d \mu \mid .
\end{aligned}
$$

Taking $c_{M}=c_{0}+c_{1} \mu^{1 / 2}(T)$, then

$$
E\left|\int_{T} g d M\right| \leq c_{M}\left(\int_{T} g^{2} d \mu\right)^{1 / 2}
$$

Proposition 3.3 If $g$ is $M$-integrable, $x^{\prime} \in X^{\prime}$, then $x^{\prime}(g)$ is $M$-integrable, and

$$
x^{\prime}\left(\int_{A} g d M\right)=\int_{A} x^{\prime}(g) d M
$$

Proof. The $M$-integrability implies that there exists a sequence $\left\{g_{k}\right\}$ of simple functions from $T$ to $X$ such that

$$
\lim _{k \rightarrow \infty} g_{k}(s)=g(s), \mu \text {-a.e. on } T
$$

and

$$
\int_{T} g d M=P-\lim _{k \rightarrow \infty} \int_{T} g_{k} d M
$$

Noting that $g_{k}$ can be written as

$$
g_{k}(s)=\sum_{n=1}^{n(k)} x_{n} \chi_{B_{n, k}}
$$

where $\chi_{B_{n, k}}$ is the characteristic function of the set $B_{n, k}$, i.e.,

$$
\chi_{n, k}(s)=\left\{\begin{array}{cc}
1 & s \in B_{n, k} \\
0, & \text { otherwise }
\end{array}\right.
$$

Therefore

$$
x^{\prime}\left(g_{k}(s)\right)=\sum_{n=1}^{n(k)} x^{\prime}\left(x_{n}\right) \chi_{B_{n, k}}
$$

Hence

$$
\lim _{k \rightarrow \infty} x^{\prime}\left(g_{k}(s)\right)=\lim _{k \rightarrow \infty} \sum_{n=1}^{n(k)} x^{\prime}\left(x_{n}\right) \chi_{B_{n, k}}=x^{\prime}\left(g_{k}(s)\right)
$$

$\mu$-a.e. on $T$. And

$$
\begin{aligned}
& P-\lim _{k \rightarrow \infty} \int_{T} x^{\prime}\left(g_{k}\right) d M \\
& =P-\lim _{k \rightarrow \infty} \sum_{n=1}^{n(k)} x^{\prime}\left(x_{n}\right) M\left(B_{n, k}\right) \\
& =x^{\prime}\left(P-\lim _{k \rightarrow \infty} \sum_{n=1}^{n(k)} x_{n} M\left(B_{n, k}\right)\right) \\
& =x^{\prime}\left(P-\lim _{k \rightarrow \infty} \int_{T} g_{k} d M\right) \\
& =x^{\prime}\left(\int_{T} g d M\right) .
\end{aligned}
$$


Consequently, $x^{\prime}(g)$ is $M$-integrable. And by the uniqueness of the limit one concludes that

$$
x^{\prime}\left(\int_{A} g d M\right)=\int_{A} x^{\prime}(g) d M
$$

\section{Limit Theorems}

This section devotes to the proofs of some limit theorems of integrals with respect to vector random measures.

A sequence $\left\{x_{k}\right\} \subset X$ is said to be weakly convergent to $x \in X$ if for every $x^{\prime} \in X^{\prime}$, there holds $\lim _{k \rightarrow \infty} x^{\prime}\left(x_{k}\right)=x^{\prime}(x)$, and denoted by $w-\lim _{k \rightarrow \infty} x_{k}=x$.

Definition 4.1 A probability measure $\mu$ is said to be a control measure of the random vector measure $M$, and denoted by $M \ll \mu$, if $\mu(A)=0$ implies $M(A)=0$.

Definition 4.2 A sequence $\left\{M_{k}\right\}$ of random vector measures is said to be weakly converges in probability to a random vector measure $M$, denoted by $w P-\lim _{k \rightarrow \infty} M_{k}=M$, if for every $x^{\prime} \in X^{\prime}$, every $E \in \Sigma$, there holds

$$
P-\lim _{k \rightarrow \infty} x^{\prime}\left(M_{k}(E)-M(E)\right)=0
$$

Definition 4.3 A function $g: T \rightarrow X$ is said to be weakly $M$ integrable, if for every $x^{\prime} \in X^{\prime}, x^{\prime}(g)$ is $M$-integrable.

Theorem 4.1 Suppose $M \ll \mu . g, g_{k} \in \mathscr{L}_{E}(M)(k=1$, $2, \ldots)$, such that $w-\lim _{k \rightarrow \infty} g_{k}(s)=g(s) \mu$-a.e. If for any given $t>0$, and if for any $x^{\prime} \in X^{\prime}$, and any $A \in \Sigma$

$$
\lim _{\mu(A) \rightarrow 0} \sup _{k} P\left\{\left\|x^{\prime}\left(\int_{A} g_{k} d M\right)\right\|>t\right\}=0
$$

then

$$
w P-\lim _{k \rightarrow \infty} \int_{A} g_{k} d M=\int_{A} g d M
$$

Proof. From Egoroff Theorem, for any given $\varepsilon>0$, there exists $A(\varepsilon) \in \Sigma, A(\varepsilon) \subset A$ and there exists $N>0$, such that $\mu(A \backslash A(\varepsilon))<\varepsilon$, and for all $s \in A(\varepsilon)$

$$
\left\|g_{k}(s)-g(s)\right\|<\varepsilon, k>N
$$

Hence

$$
\begin{aligned}
& P\left[\left\|x^{\prime}\left(\int_{A(\varepsilon)} g_{k} d M-\int_{A(\varepsilon)} g d M\right)\right\|>t\right] \\
& =P\left[\left\|x^{\prime}\left(\int_{A(\varepsilon)} x^{\prime}\left(g_{k}-g\right) d M\right)\right\|>t\right] \\
& \leq 2 P\left\{\left\|x^{\prime}\left(g_{k}-g\right)\right\|_{\infty} \cdot\|M(T)\|\right\},
\end{aligned}
$$

Combining (5) and (6) one concludes that

$$
\lim _{k \rightarrow \infty} P\left[\left\|x^{\prime}\left(\int_{A(\varepsilon)} g_{k} d M-\int_{A(\varepsilon)} g d M\right)\right\|>t\right]=0
$$

Therefore, for any given $x^{\prime} \in X^{\prime}$

$$
\begin{aligned}
& P\left[\left\|x^{\prime}\left(\int_{A} g_{k} d M-\int_{A} g d M\right)\right\|>t\right] \\
& \leq P\left[\left\|x^{\prime}\left(\int_{A} g_{k} d M-\int_{A(\varepsilon)} g_{k} d M\right)\right\|>t\right] \\
& +P\left[\left\|x^{\prime}\left(\int_{A(\varepsilon)} g_{k} d M-\int_{A(\varepsilon)} g d M\right)\right\|>t\right] \\
& +P\left[\left\|x^{\prime}\left(\int_{A(\varepsilon)} g d M-\int_{A} g d M\right)\right\|>t\right] . \\
& =P\left[\left\|x^{\prime}\left(\int_{B(\varepsilon)} g_{k} d M\right)\right\|>t\right] \\
& +P\left[\left\|x^{\prime}\left(\int_{A(\varepsilon)} g_{k} d M-\int_{A(\varepsilon)} g d M\right)\right\|>t\right] \\
& +P\left[\left\|x^{\prime}\left(\int_{B(\varepsilon)} g d M\right)\right\|>t\right],
\end{aligned}
$$

where $B(\varepsilon)=A \backslash A(\varepsilon)$. From the assumption

$$
\begin{aligned}
& \lim _{k \rightarrow \infty} P\left[\left\|x^{\prime}\left(\int_{B(\varepsilon)} g_{k} d M\right)\right\|>t\right]=0 \\
& \lim _{k \rightarrow \infty} P\left[\left\|x^{\prime}\left(\int_{B(\varepsilon)} g d M\right)\right\|>t\right]=0
\end{aligned}
$$

Therefore

$$
w P-\lim _{k \rightarrow \infty} \int_{A} g_{k} d M=\int_{A} g d M
$$

Similar to the proof of Theorem 4.1 one has the following Theorem 4.2.

Theorem 4.2 Suppose $M \ll \mu . g, g_{k} \in \mathscr{L}_{E}(M)(k=1$, $2, \ldots)$, such that $\lim _{k \rightarrow \infty} g_{k}(s)=g(s) \mu$-a.e. For given $t>0$, if

$$
\lim _{\mu(A) \rightarrow 0} \sup _{k} P\left\{\left\|x^{\prime}\left(\int_{A} g_{k} d M\right)\right\|>t\right\}=0
$$

Then, for any $A \in \Sigma$

$$
P-\lim _{k \rightarrow \infty} \int_{A} g_{k} d M=\int_{A} g d M
$$

Theorem 4.3 Suppose $M<<\mu . g, g_{k} \in \mathscr{L}_{E}(M)(k=1$, $2, \ldots)$, such that $\lim _{k \rightarrow \infty} g_{k}(s)=g(s) \mu$-a.e. If there exists $C>0$ such that $\left\|g_{k}(s)\right\| \leq C,(n=1,2, \ldots)$, then $g$ is $M$-integrable, and for every $A \in \Sigma$

$$
P-\lim _{k \rightarrow \infty} \int_{A} g_{k} d M=\int_{A} g d M .
$$

Proof. Since $M<<\mu$,

$$
\begin{aligned}
& \lim _{\mu(A) \rightarrow 0} \sup _{k} P\left[\left\|\int_{A} g_{k} d M\right\|>t\right] \\
& \leq \lim _{\mu(A) \rightarrow 0} \sup _{k} 2 P\left[\left\|g_{k}\right\|_{\infty} \cdot\left\|\int_{A} 1 d M\right\|>t\right] \\
& \leq \lim _{\mu(A) \rightarrow 0} \sup _{k} 2 P[C \cdot\|M(A)\|>t] \\
& =0 .
\end{aligned}
$$

Therefore, from Theorem 4.2

$$
P-\lim _{k \rightarrow \infty} \int_{A} g_{k} d M=\int_{A} g d M
$$


Theorem 4.4 Suppose $M, M_{k}(k=1,2, \ldots)$ are random vector measures, and $P-\lim _{k \rightarrow \infty} M_{k}=M, g \in \mathscr{L}_{E}(M)$ and $g$ is a bounded function under the weak topology of $X$, then $g$ is weakly $M$-integrable and for every $A \in \Sigma$

$$
w P-\lim _{k \rightarrow \infty} \int_{A} g d M_{n}=\int_{A} g d M
$$

Proof. From Proposition 2.3, $g$ is weakly $M$-integrable. Noting that $P-\lim _{k \rightarrow \infty} M_{k}=M$

$$
\begin{aligned}
& P\left[\left\|x^{\prime}\left(\int_{A} g d M_{k}-\int_{A} g d M\right)\right\|>t\right] \\
& \leq P\left[\left\|\int_{A} x^{\prime} g\left(d M_{k}-d M\right)\right\|>t\right] \\
& \leq 2 P\left[\left\|x^{\prime}(g)\right\|_{\infty} \cdot\left\|\left(M_{k}-M\right)(T)\right\|>t\right] \\
& +P\left[\left\|x^{\prime}\left(\int_{A(\varepsilon)} g d M-\int_{A} g d M\right)\right\|>t\right] . \\
& =0 .
\end{aligned}
$$

This completes the proof.

Theorem 4.5 Suppose $M, M_{k}(k=1,2, \ldots)$ are random vector measures, where $M_{k} \cong\left[\sigma^{2}, \mu_{n}\right]$, and $E\left|M_{k}(T)\right|<\infty(k$ $=1,2, \ldots), g$ is a bounded continuous function. If $\left\{\mu_{k}\right\}$ weakly converges to $\mu$, and if $\left\{M_{k}\right\}$ weakly converges to $M$, then

$$
w P-\lim _{k \rightarrow \infty} \int_{T} g d M_{n}=\int_{T} g d M
$$

Proof. For every $n>0$, there exists a compact subset $B \subset T$ and a constant $c>0$ such that

$$
\sup _{k} \mu_{k}(B)<c<\infty, \sup _{k} \mu_{k}(T \backslash B)<\frac{1}{n},
$$

and there exists a sequence of simple function

$$
g_{l}(s)=\sum_{n=1}^{n(l)} x_{n} \chi_{B, l}(l=1,2, \ldots)
$$

such that

$$
\left\|g_{l}(s)-g(s)\right\|<\frac{1}{n}, s \in B
$$

and

$$
\sup \left\|g_{l}(s)\right\| \leq \sup \|g(s)\|<c_{0}(l=1,2, \ldots) .
$$

Therefore

$$
\begin{aligned}
& \int_{T}\left\|g-g_{l}\right\|^{2} d \mu_{n} \\
& =\int_{B}\left\|g-g_{l}\right\|^{2} d \mu_{k}+\int_{T \backslash B}\left\|g-g_{l}\right\|^{2} d \mu_{k} \\
& \leq n^{-2} \mu_{n}(B)+\left(2 c_{0}\right)^{2} \mu_{n}(T \backslash B) \\
& \leq n^{-2} c+\left(2 c_{0}\right)^{2} n^{-1} .
\end{aligned}
$$

Hence, there exists a constant $c_{1}>0$ such that

$$
\begin{aligned}
& E\left\|\int_{T} g_{l} d M_{k}-\int_{T} g d M_{k}\right\| \\
& \leq c_{1}\left(m^{-2} c+\left(2 c_{0}\right)^{2} n^{-1}\right)^{\frac{1}{2}},
\end{aligned}
$$

By Chebyshev's Inequality, for every $t>0$

$$
\lim _{l \rightarrow \infty} \limsup _{k} P\left\{\left\|\int_{T} g_{l} d M_{k}-\int_{T} g d M_{k}\right\|>t\right\}=0
$$

Similarly,

$$
\lim _{l \rightarrow \infty} P\left\{\left\|\int_{T} g_{l} d M-\int_{T} g d M\right\|>t\right\}=0
$$

Noting that

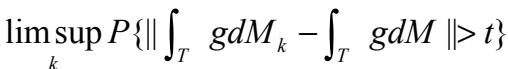

$$
\begin{aligned}
& \leq \underset{k}{\limsup } P\left\{\left\|\int_{T} g d M_{k}-\int_{T} g_{l} d M_{k}\right\|>t\right\}
\end{aligned}
$$

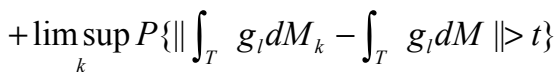

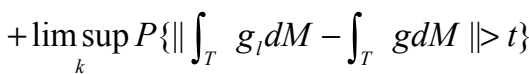

$$
\begin{aligned}
& =\underset{k}{\limsup } P\left\{\left\|\int_{T} g_{l} d M_{k}-\int_{T} g_{l} d M\right\|>t\right\}
\end{aligned}
$$

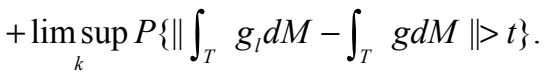

Hence

$$
\lim _{k \rightarrow \infty} P\left\{\left\|\int_{T} g d M_{k}-\int_{T} g d M\right\|>t\right\}=0
$$

That is to say

$$
P-\lim _{k \rightarrow \infty} \int_{T} g d M_{n}=\int_{T} g d M
$$

On the other hand, for any $A_{n} \in \Sigma(n=1,2, \ldots)$ satisfying $\lim _{n \rightarrow \infty} \mu\left(A_{n}\right)=0$ there exists a constant $c_{2}>0$ such that

$$
E\left\|\int_{A_{n}} g d M_{k}\right\| \leq c_{2}\left(\int_{A_{n}}\|g\|^{2} d \mu_{k}\right)^{\frac{1}{2}}
$$

Since $\left\{\mu_{k}\right\}$ weakly converges to $\mu$,

$$
\lim _{n \rightarrow \infty} \limsup \int_{A_{n}}\|g\|^{2} d \mu_{k} \leq \int_{A_{n}}\|g\|^{2} d \mu
$$

Hence

$$
\lim _{n \rightarrow \infty} \limsup _{k} E\left\|\int_{A_{n}} g d M_{k}\right\|=0
$$

Again, Chebyshev's Inequality implies that

$$
\lim _{n \rightarrow \infty} \limsup _{k} P\left\{\left\|\int_{A_{n}} g d M_{k}\right\|>t\right\}=0
$$

Which completes the proof. 


\section{Conclusion Remark}

Let $(T, \Sigma)$ be a measurable space. A countably additive set function on $\Sigma$ and taking values in $L_{0}(\Omega, P)$ is said to be a random measure. Vector random measures arise naturally as a "Banach space generalization" of real-valued random measures. Let $X$ be a Banach space, $L_{0}^{X}(\Omega, P)$ the set of all $X$-valued random variables. A countably additive set function on $\Sigma$ taking values in $L_{0}^{X}(\Omega, P)$ is said to be an $X$-valued random measure. Vector random measures can be regarded as a "natural random generalization" of vector non-random measures studied by many authors.

This paper extends the definitions of random measure and random integral to complete paranormed spaces, and devotes to the study of limit theorems of random integrals with respect to vector random measures.

\section{References}

[1] S. Bochner, Stochastic Process, Annals of Mathematics, (48)1947(1), 1014-1061.

[2] F. Treves, Topological Vector Spaces, Distribution and Kernels, Academic Press, 1967.

[3] J. Rosinski, Random Integrals of Banach Space Valued Functions, Studia Mathematica, 8(1984), 15-38.

[4] P. Billingsley, Convergence of Probability Measures, John Wiley \& Son, Inc. 1968.

[5] R. Zeng, the Completeness of $L_{X}^{1}(\mu, \Sigma)$, Journal of Mathematical Research with Applications, 15(1995)5, 40-48.

[6] L. Egghe, The Radon-Nikodym Property, densibility and Martingles in Loccally Convex Space, Pacific Journal of Mathematics, 87(1980)2, 313-322.

[7] D. H. Thang, On the Convergence of Vector Random Measures, Probability Theory and Related Fields, 88(1991), 116.

[8] A. N. Baushev, On the Weak Convergence of Probability Measures in Orlicz Spaces, Theory of Probability and Applications, 40(1996)3, 420-429.

[9] R. Jajte and W. D. A. Paszkiewicz, Probability and Mathematical Statistics Conditioning and Weak Convergence,
Probability and Mathematical Statistics, 19(1999)2, 453-461.

[10] N. Guillotin-Plantard and V. Ladret, Limit Theorems for Ustatistics Indexed by a One Dimensional Random Walk, ESAIM: Probability and Statistics, 9(2005), 98-115.

[11] H. Tsukahara, On the Convergence of Measurable Processes and Prediction Processes, Illinois Journal of Mathematics, 51(2007)4, 1231-1242.

[12] X. F. Yang, Integral Convergence Related to Weak Convergence of Measures, Applied Mathematical Sciences, 56(2011)5, $2775-2779$.

[13] T. Grbic and S. Medic, Weak Convergence of Sequences of Distorted Probabilities, SISY 2015, Proceedings of IEEE $13^{\text {th }}$ International Symposium on Intelligent System and Informatics, Sept. 2015, 307-318, Subotica, Serbia.

[14] T. E. Govindan, Weak Convergence of Probability Measures of Yosida Approximate Mild Solutions of McKean-Vlasov Type Stochastic Evolution Equations, Seventh International Conference on Dynamic Systems and Applications \& Fifth International Conference on Neural, Parallel, and Scientific Computations, May 2015, Atlanta, USA.

[15] P. Puchala, Weak Convergence in $L^{1}$ of the Sequences of Monotonic Functions, Journal of Applied Mathematics and Computational Mechanics 13(2014)3, 195-199.

[16] L. Meziani, A Theorem of Riesz Type with Pettis Integrals in Topological Vector Spaces, Journal of Mathematical Analysis and Applications, 340 (2008), 817-824.

[17] F. J. Pinski, G. Simpson, A. M. Stuart and H. Weber, Kullback-Leibler Ppproximation for Probability Measures on Infinite Dimensional Spaces, SIAM Journal on Mathematical Analysis, 47(2015)6, 4091-4122.

[18] W. Lohrl and T. Ripple, Boundedly Finite Measures: Separation and Convergence by an Algebra of Functions, Electronic Communication of Probability, 21(2016)60, 1-16.

[19] H. H. Wei, Weak Convergence of Probability Measures on Metric Spaces of Nonlinear Operators, Bulletin of the Institute of Mathematics Academia Sinica, 11(2016)3, 485-519.

[20] D. Dolgopyat, A Local Limit Theorem for Sums of Independent Random Vectors, Electronic Journal of Probability, 39(2017)1-15.

[21] G. Naitzat and R. J. Adler, A Central Limit Theorem for the Euler Integral of a Gaussian random field, Stochastic Processes and Their Applications, 127(2017)6, 2036-2067. 